\title{
ChemComm
}

\section{Synthesis of monodisperse organosilica nanoparticles with hollow interiors and porous shells using silica nanospheres as templates $\dagger$}

Cite this: Chem. Commun., 2013, 49, 4998

Received 14th March 2013, Accepted 9th April 2013

DOI: $10.1039 /$ c3cc41904e

www.rsc.org/chemcomm

A versatile method for the formation of monodisperse, bridged silsesquioxane nanoparticles with hollow interiors and porous shells has been developed using silica nanospheres as templates. Tunable size and shell thickness, as well as high surface areas and large pore volumes of the hollow particles, allow for practical application of these nanoparticles in many fields.

Porous silica nanoparticles have numerous potential applications including in catalysis, ${ }^{1}$ bio-sensing, ${ }^{2}$ drug delivery ${ }^{3,4}$ and anti-reflection (AR) coatings. ${ }^{5,6}$ High dispersibility as well as precisely controlled particle size are crucial for these applications. Additionally, hollow structures with porous shells are important, for example, for enhancing drug storage capacity and decreasing the refractive index of AR coatings. Bridged silsesquioxanes (hereafter denoted organosilica), prepared by hydrolysis and polycondensation of precursors with the general formula $\left(\mathrm{R}^{\prime} \mathrm{O}\right)_{3} \mathrm{Si}-\mathrm{R}-\mathrm{Si}\left(\mathrm{OR}^{\prime}\right)_{3}\left(\mathrm{R}, \mathrm{R}^{\prime}=\right.$ organic groups), have several advantages as shell components, including facile functionalization and potential to modify mechanical and physical properties of the particles. ${ }^{7-10}$ Hollow organosilica nanoparticles have recently been achieved using block copolymer micelles as soft templates; ${ }^{11-13}$ however, such a method generally requires careful adjustments of the molecular weight of the polymers as well as synthesis conditions for fine-tuning the interior size of the particles.

Here we report a new method for preparing monodisperse, hollow organosilica nanoparticles using silica nanospheres (SNSs) ${ }^{14,15}$ as hard templates (Fig. 1A). A shell comprising tiny particles of organosilica was successfully formed on SNSs by stirring a biphasic mixture of organoalkoxysilane precursors and an aqueous dispersion of SNSs. Interparticle mesopores within the shell allowed the removal of the internal silica core; this is attributed to the much lower hydrolytic stability of pure silica compared to organosilica ${ }^{10}$ under elevated $\mathrm{pH}$ conditions and is in clear contrast to conventional syntheses of hollow

Department of Chemical System Engineering, The University of Tokyo, 7-3-1 Hongo, Bunkyo-ku, Tokyo 113-8656, Japan. E-mail: okubo@chemsys.t.u-tokyo.ac.jp; Fax: +81-3-5800-3806; Tel: +81-3-5841-7348

$\dagger$ Electronic supplementary information (ESI) available: Experimental details and Fig. S1-S9. See DOI: 10.1039/c3cc41904e

‡ Present address: Department of Applied Chemistry, Waseda University, 3-4-1 Ohkubo, Shinjuku-ku, Tokyo 169-8555, Japan.

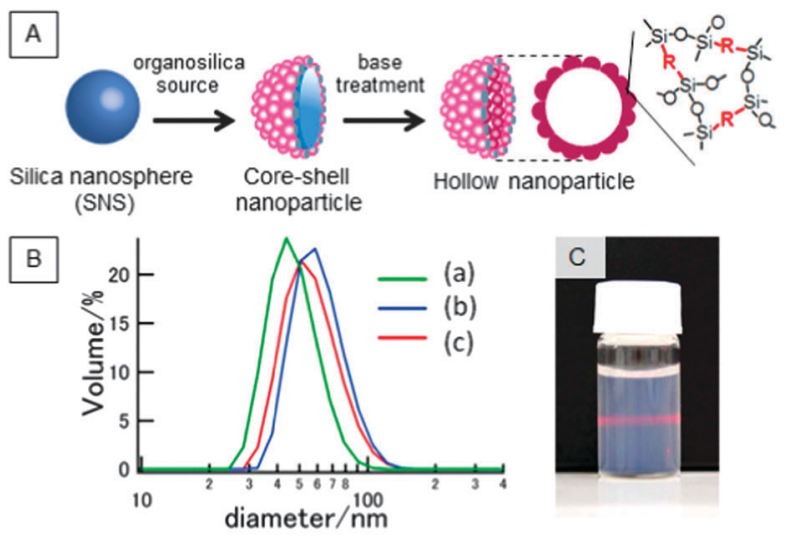

Fig. 1 (A) Schematic of the formation of hollow organosilica nanoparticles using SNSs as templates. (B) Dynamic light scattering curves of the dispersions of (a) SNS sized ca. $40 \mathrm{~nm}$, (b) SNS core-organosilica ( $\mathrm{R}=-\mathrm{CH}_{2}-\mathrm{CH}_{2}-$ ) shell nanoparticles and (c) hollow organosilica nanoparticles. (C) Photograph of the dispersion of hollow organosilica nanoparticles (ca. $1.2 \mathrm{wt} \%$ ).

mesoporous silica nanoparticles ${ }^{16-18}$ where surfactant templates are employed to form porous shells. Thus, a low-cost and efficient route to monodisperse, hollow organosilica nanoparticles with narrow size distributions has been established.

In a typical procedure, monodisperse SNSs sized $c a .40 \mathrm{~nm}$ were prepared in a biphasic system using arginine as a base catalyst (see $\mathrm{ESI} \dagger$ for details). To this dispersion (ca. $6.3 \mathrm{wt} \% \mathrm{SiO}_{2}, 5 \mathrm{~mL}$ ), additional water $(20 \mathrm{~mL})$, L-arginine (0.02 g) and 1,2-bis(triethoxysilyl)ethane (BTEE, Aldrich, $0.8 \mathrm{~g}$ ) were added, and the biphasic mixture was stirred at $60{ }^{\circ} \mathrm{C}$ until the upper oil phase (BTEE) disappeared (over several days) to form core-shell nanoparticles. The $\mathrm{pH}$ of the dispersion was then increased to 13 by adding $0.1 \mathrm{~N} \mathrm{NaOH}$. After stirring at room temperature for 2 days, the dispersion was dialyzed against deionized water for 1 day to obtain a clear dispersion of hollow organosilica nanoparticles. Dynamic light scattering (Fig. 1B) shows that the particles are monodispersed throughout these procedures and that the particle size increases after shell formation and slightly decreases after the core removal. Importantly, the hollow nanoparticle dispersion is quite stable; no precipitate is observed even after 1 month (Fig. 1C). This is attributed to strong electrostatic 

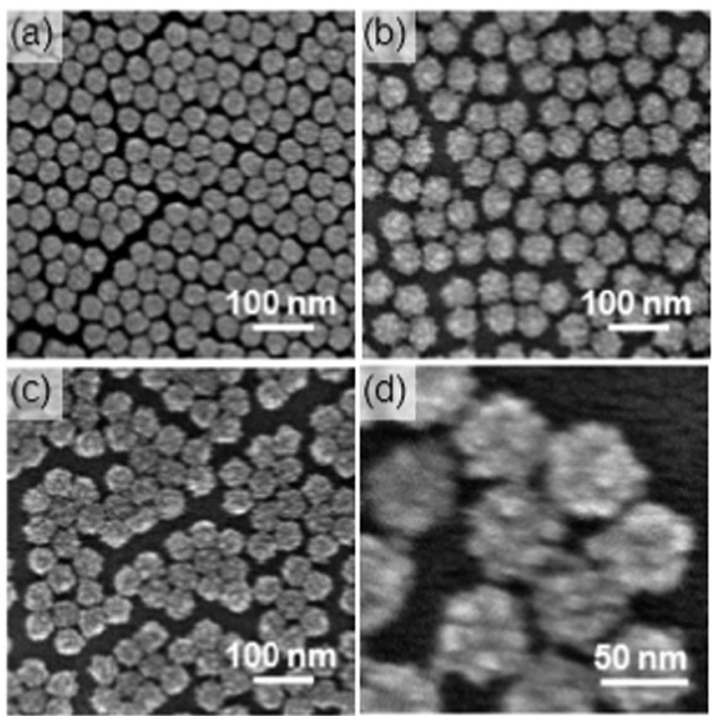

(e)

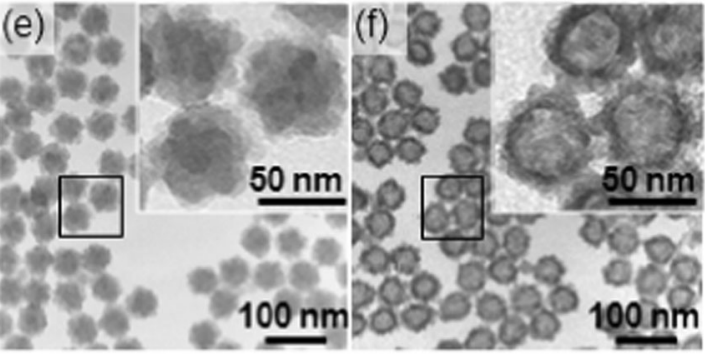

Fig. 2 FE-SEM images of (a) SNSs ca. $40 \mathrm{~nm}$ in diameter, (b) core-shell nanoparticles and ( $c$ and $d$ ) hollow organosilica nanoparticles. TEM images of (e) core-shell nanoparticles and (f) hollow organosilica nanoparticles. The insets show enlarged images of the selected areas.

repulsion between the particles, as suggested by the high absolute value of the zeta potential $(-34 \mathrm{mV}$ at $\mathrm{pH} 7.8)$.

Fig. 2(a)-(d) show the field-emission scanning electron microscopy (FE-SEM) images of (a) SNSs ca. $40 \mathrm{~nm}$ in diameter, (b) core-shell nanoparticles and (c and d) hollow organosilica nanoparticles obtained by the dissolution of the SNS core. The core-shell and hollow nanoparticles both retained the narrow size distribution particular to SNSs. The core-shell particles have a characteristic, granular shell consisting of tiny particles $5-10 \mathrm{~nm}$ in size; some interparticle mesopores are also observed on the shell. Similar shell morphology is preserved for the hollow organosilica nanoparticles. The morphology was retained even after sonication (Fig. S1 in ESI + ), suggesting that the organosilica particles that constitute the shell are tightly attached to each other by $\mathrm{Si}-\mathrm{O}-\mathrm{Si}$ bonds. Because of the high dispersibility and uniform size and shape, an ordered monolayer film of the hollow particles can be produced by spin-coating the dispersion onto a silicon substrate (Fig. S2 in ESI + ). This property is particularly important for thin film applications.

Transmission electron microscopy (TEM) images of the core-shell particles and hollow organosilica nanoparticles are shown in Fig. 2(e) and (f), respectively. The core-shell particles turned into hollow structures after the $\mathrm{pH}$ was increased. The size of the interior space was slightly smaller than that of the SNS template, suggesting that shell shrinkage occurred upon core removal. In contrast to the irregular morphology of the external surface of the shell, the internal surface was smooth, reflecting the spherical morphology of the SNSs.

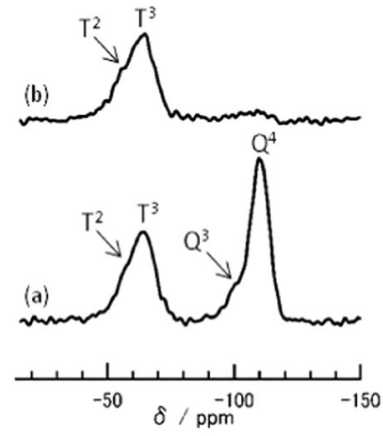

Fig. 3 Solid-state ${ }^{29}$ Si MAS NMR spectra of (a) core-shell nanoparticles and (b) hollow organosilica nanoparticles prepared using SNSs ca. $40 \mathrm{~nm}$ in diameter.

Quantitative information about the removal of the SNS core was obtained using solid-state ${ }^{29}$ Si MAS NMR (Fig. 3). The spectrum of the core-shell nanoparticles shows the $\mathrm{T}^{2}$ and $\mathrm{T}^{3}$ signals arising from the organosilica shell, in addition to the $\mathrm{Q}^{3}$ and $\mathrm{Q}^{4}$ signals of the SNSs $\left(\mathrm{T}^{m}\right.$ and $\mathrm{Q}^{n}$ represent $\mathrm{CSi}(\mathrm{OSi})_{m}(\mathrm{OH})_{3-m}$ and $\mathrm{Si}(\mathrm{OSi})_{n}(\mathrm{OH})_{4-n}$, respectively). The integral intensity ratio of $\left(\mathrm{Q}^{3}+\mathrm{Q}^{4}\right) /\left(\mathrm{T}^{2}+\mathrm{T}^{3}\right)$ is 1.4 for the core-shell nanoparticles, which greatly decreased to 0.09 for the hollow nanoparticles, meaning that $c a$. $93 \%$ of the $\mathrm{SiO}_{4}$ species was removed. It is known that hydrolysis of Si-O-Si proceeds via nucleophilic attack of $\mathrm{HO}^{-}$on the $\mathrm{Si}$ atom; therefore, higher electron density of $\mathrm{Si}$ in organosilica can lead to a lower dissolution rate. FT-IR analysis suggested that $\mathrm{Si}-\mathrm{C}$ cleavage did not occur upon core removal (Fig. S3 in ESI $\dagger$ ).

Fig. 4A compares the nitrogen adsorption-desorption isotherms of (a) SNSs (ca. $40 \mathrm{~nm}$ ), (b) core-shell nanoparticles and (c) hollow organosilica nanoparticles. All of the isotherms can be classified as IUPAC type IV, characteristic of mesoporous solids. The total pore volume increased from $0.25 \mathrm{~cm}^{3} \mathrm{~g}^{-1}$ to $0.46 \mathrm{~cm}^{3} \mathrm{~g}^{-1}$ as a result of shell formation and increased further to $1.45 \mathrm{~cm}^{3} \mathrm{~g}^{-1}$ when the cores were removed. The Brunauer-Emmett-Teller (BET) specific surface area of the core-shell nanoparticles $\left(208 \mathrm{~m}^{2} \mathrm{~g}^{-1}\right)$ was higher than that of the SNSs $\left(118 \mathrm{~m}^{2} \mathrm{~g}^{-1}\right)$, which can be attributed to the irregular surface morphology of the shell. The BET surface area was further enhanced to $530 \mathrm{~m}^{2} \mathrm{~g}^{-1}$ after the core removal. The Barrett-Joyner-Halenda (BJH) pore size distribution of the hollow nanoparticles shows two peaks: one centred at $33 \mathrm{~nm}$ and a relatively broad peak in the range of 6 to $30 \mathrm{~nm}$ (Fig. 4B). The former corresponds to the internal space of the particles. The latter peak can be ascribed to interparticle pores because a

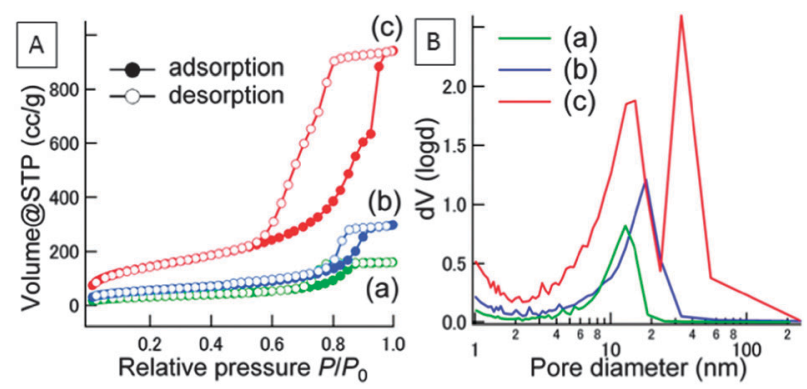

Fig. 4 (A) Nitrogen adsorption-desorption isotherms and (B) BJH pore size distribution curves of (a) SNS ca. $40 \mathrm{~nm}$ in diameter, (b) core-shell nanoparticles and (c) hollow organosilica nanoparticles. 


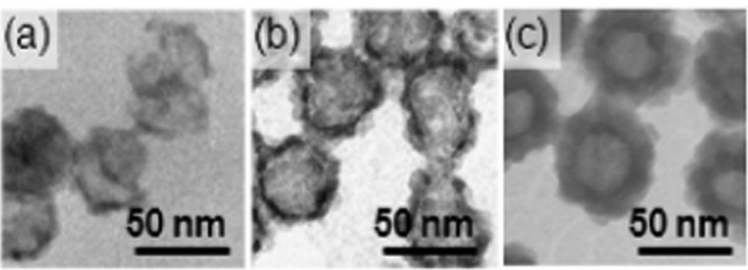

Fig. 5 TEM images of hollow organosilica nanoparticles synthesized with varying amounts of BTEE under otherwise identical conditions: (a) $0.29 \mathrm{~g}$, (b) $0.60 \mathrm{~g}$ and (c) $2.2 \mathrm{~g}$.

similar peak was observed for both SNSs and the core-shell nanoparticles.

The granular morphology of the organosilica shell is different from the relatively smooth surface morphologies formed by adding TEOS to the dispersion of SNSs. ${ }^{15}$ In both cases, seed growth is more dominant than the formation of new particles, which can be explained by the slow hydrolysis rates of alkoxysilanes reacting at the oil-water interface under mild basic conditions. The difference in the shell morphologies is probably due to the much lower solubility of organosilica $\left(\mathrm{O}_{1.5} \mathrm{Si}-\mathrm{R}-\mathrm{SiO}_{1.5}\right)$ compared with silica $\left(\mathrm{SiO}_{2}\right)$. During the formation of the silica shell, ${ }^{15}$ partial dissolution and re-deposition on the particle surface occur concurrently, which leads to smooth surfaces with lower energy. In contrast, organosilica is hardly dissolved during the shell formation; hence, the primary particles deposited on the SNSs in the early stage of the reaction grow continuously to form a granular shell. In the absence of SNSs, granular particles without hollow interiors were formed (Fig. S4 in ESI†). The size of the primary particles was similar to that observed for the hollow nanoparticles.

The thickness of the organosilica shell can be easily controlled by varying the amount of BTEE (Fig. 5 and Fig. S5 in ESI†). The sizes of the core-shell and hollow nanoparticles increase with increasing amounts of BTEE while maintaining the monodispersity. It appears that the size of the shell-forming particles increases with increasing BTEE amount, which is consistent with the aforementioned formation mechanism. When the amount of BTEE was too small, partial collapse of the shell occurred upon core removal (Fig. 5(a)).

To demonstrate the wide applicability of the presented method, SNSs of different sizes have been used as templates. Hollow nanoparticles with similar shell morphologies but larger cavities were obtained using SNSs sized $c a .60 \mathrm{~nm}$ (Fig. S6 in ESI†). Also, the bridging organic group in the shell is not limited to ethylene $\left(-\mathrm{CH}_{2}-\mathrm{CH}_{2}^{-}\right)$; other bridged organoalkoxysilanes can be used instead of BTEE. For example, we successfully synthesised hollow organosilica nanoparticles using 1,4-bis(triethoxysilyl)benzene (Fig. S7 in ESI + ). This is important for functionalization and for tuning the properties of the shell.

Improvements to this method have also been made with a view towards a simpler and lower-cost synthesis. From the industrial viewpoint, arginine should be replaced with more common base catalysts such as $\mathrm{NaOH}$ and $\mathrm{NH}_{4} \mathrm{OH}$. We confirmed that $\mathrm{NaOH}$ can be used not only for the synthesis of SNSs, but also for the formation of the organosilica shell, producing hollow organosilica nanoparticles (Fig. S8 in ESI $\dagger$ ). This result implies that a wide variety of colloidal silica nanoparticles can be used as templates.
On the other hand, a preliminary experiment showed that one-step synthesis of core-shell nanoparticles is possible. When starting from an oil-water biphasic mixture where the oil phase consists of TEOS and BTEE, preferential hydrolysis of TEOS is confirmed using ${ }^{13} \mathrm{C}$ NMR (Fig. S9 in ESI + ). After treatment at high pH, hollow nanoparticles were observed, although the removal of the cores was not complete. Efforts are underway to optimize the synthesis conditions.

In conclusion, an efficient, versatile route to monodisperse and size-tunable organosilica nanoparticles with hollow interiors has been achieved using silica nanospheres as hard templates. Porous shells are formed from the biphasic mixtures of bridged organoalkoxysilane and aqueous dispersions of SNSs, allowing removal of the cores through interparticle voids. Narrow size distributions, high porosity, and water dispersibility of these hollow nanoparticles make them promising candidates for applications in low- $k /$ low- $n$ coatings and drug delivery systems.

We thank Prof. Yukio Yamaguchi (The University of Tokyo) for zeta potential and dynamic light scattering measurements. This work was supported in part by a Grant-in-Aid for Scientific Research (B) and a Grant-in Aid for Scientific Research on the Innovative Areas: 'Fusion Materials' (Area No. 2206) from Ministry of Education, Culture, Sports, Science and Technology (MEXT), Japan. A part of this work was conducted at Center for Nano Lithography \& Analysis, The University of Tokyo, supported by MEXT.

\section{Notes and references}

1 K. C.-W. Wu and Y. Yamauchi, J. Mater. Chem., 2012, 22, 1251.

2 I. I. Slowing, B. G. Trewyn, S. Giri and V. S.-Y. Lin, Adv. Funct. Mater., 2007, 17, 1225.

3 F. Tang, L. Li and D. Chen, Adv. Mater., 2012, 24, 1504.

4 P. Yang, S. Gai and J. Lin, Chem. Soc. Rev., 2012, 41, 3679.

5 M. Yamaguchi, H. Nakayama, K. Yamada and H. Imai, Opt. Lett., 2009, 34, 2060.

6 Y. Hoshikawa, H. Yabe, A. Nomura, T. Yamaki, A. Shimojima and T. Okubo, Chem. Mater., 2010, 22, 12.

7 F. Hoffmann, M. Cornelius, J. Morell and M. Fröba, Angew. Chem., Int. Ed., 2006, 45, 3216.

8 B. P. Pichon, M. Wong Chi Man, P. Dieudonné, J.-L. Bantignies, C. Bied, J.-L. Sauvajol and J. J. E. Moreau, Adv. Funct. Mater., 2007, 17, 2349.

9 Y. Lu, H. Fan, N. Doke, D. A. Loy, R. A. Assink, D. A. LaVan and C. J. Brinker, J. Am. Chem. Soc., 2000, 122, 5258.

10 C. Urata, H. Yamada, R. Wakabayashi, Y. Aoyama, S. Hirosawa, S. Arai, S. Takeoka, Y. Yamauchi and K. Kuroda, J. Am. Chem. Soc., 2011, 133, 8102.

11 (a) J. Liu, Q. Yang, L. Zhang, H. Yang, J. Gao and C. Li, Chem. Mater., 2008, 20, 4268; (b) J. Liu, S. Bai, H. Zhong, C. Li and Q. Yang, J. Phys. Chem. C, 2010, 114, 953.

12 M. Mandal and M. Kruk, Chem. Mater., 2012, 24, 123.

13 M. Sasidharan, K. Nakashima, N. Gunawardhana, T. Yokoi, M. Ito, M. Inoue, S. Yusa, M. Yoshio and T. Tatsumi, Nanoscale, 2011, 3, 4768.

14 T. Yokoi, Y. Sakamoto, O. Terasaki, Y. Kubota, T. Okubo and T. Tatsumi, J. Am. Chem. Soc., 2006, 128, 13664.

15 T. Yokoi, J. Wakabayashi, Y. Otsuka, W. Fan, M. Iwama, R. Watanabe, K. Aramaki, A. Shimojima, T. Tatsumi and T. Okubo, Chem. Mater., 2009, 21, 3719.

16 Y. Chen, H. Chen, L. Guo, Q. He, F. Chen, J. Zhou, J. Feng and J. Shi, ACS Nano, 2010, 4, 529.

17 Y. Jiao, J. Guo, S. Shen, B. Chang, Y. Zhang, Y. Zhang, X. Jiang and W. Yang, J. Mater. Chem., 2012, 22, 17636.

18 T. Ikuno, A. Nomura, K. Iyoki, A. Sugawara-Narutaki, T. Okubo and A. Shimojima, Chem. Lett., 2013, 316. 